\title{
Simulation and contrast on three kinds of guidance law of guided bomb
}

\author{
Qing Zhang ${ }^{1}$, Yongshan Liu $^{1}$ \\ ${ }^{1}$ School of Aerospace Engineering, Beijing Institute of Technology, Beijing 100081, China; \\ ${ }^{1}$ School of Aerospace Engineering, Beijing Institute of Technology, Beijing 100081, China. \\ ajK8326@outlook.com, ${ }^{b}$ liuysh@bit.edu.cn
}

Keywords: guided-bomb, guidance law, simulation, contrast.

\begin{abstract}
Firstly, guidance law is proposed in order to enhance the performance of the guided-bomb. Secondly, the gravity PN guidance law, bias proportional navigation guidance law with terminal impact angle constraint and sliding mode variable structure guidance law with terminal impact angular constraint are all derived, simulated and analyzed in the terminal stage. Finally, contrast on the performance is conducted among the three kind of guidance law.
\end{abstract}

\section{Introduction}

The guidance loop is both the outside loop of aviation guided-bomb and a measuring mechanism which generally installed in the projectile body or the ground. The measuring mechanism is generally refers to GPS or Inertial Device or seeker which can observe and detect the instant signal of the target and the projectile body. In response to the signal, instant guiding instruction is formed based on the aiming means and then transmitted to the guiding system so that the guided-bomb along the path to ruin the target efficiently.

Based on the low muzzle velocity and low overload features of low-speed launched guided-bomb, ballistic trajectory is generally partitioned to four parts: uncontrolled stage, middle stage, handing-off stage and terminal stage. The terminal stage is from the point which handing-off stage ends to the point that the guided-bomb and the target meet. This stage is quite critical because it influence the ultimate performance of the guided-bomb.

The index that reflects the performance of the terminal stage of the guided-bomb mainly includes landing error and impact angle constraint. To strengthen the penetration effect of the guided-bomb against enemy target. Only through decreasing the landing error and increasing impact angle constraint that can be achieved by improved guidance law in the terminal stage [1].

\section{Interactive movement model}

The interactive movement illustration and equation set between the target and the guided-bomb are needed when aiming means is applied to engineering field. According to the illustration and the equation set, we can get all the information about the target and the guided-bomb [2].

Figure 1 illustrates the guidance geometry composed of these variables. Here, $q$ refers to line-of-sight angle, $\theta$ to path angle of guided-bomb, $\theta_{t}$ to course angle of target, $\eta$ to velocity vector lead angle of guided-bomb, $\eta_{t}$ to velocity vector lead angle of target, $N_{t}$ to overload of target, $N_{c}$ to overload of instruction of guidance law.

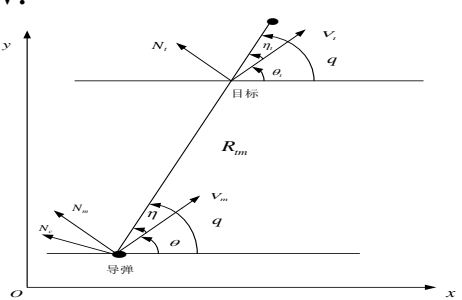

Fig. 1 Guidance geometry of the target and the guided-bomb

Based on Fig 1, list the equation set of geometrical relationship of these variables.. 


$$
\begin{aligned}
& \frac{d R_{t m}}{d t}=V_{t} \cos \eta_{t}-V \cos \eta \\
& R_{t m} \frac{d q}{d t}=V \sin \eta-V_{t} \sin \eta_{t} \\
& q=\theta+\eta \\
& q=\theta_{t}+\eta_{t} \\
& V_{m} \dot{\theta}=N_{c} \\
& V_{t} \dot{\theta}_{t}=N_{t}
\end{aligned}
$$

Line-of-sight velocity between target and guide-bomb can't be measured directly, it is deduced by related arithmetic during which velocity and position of guided-bomb and target are needed.

Based on Fig 1, list the differential coefficient equation set.

$$
\left.\begin{array}{l}
\dot{q}=\frac{R_{t m x} V_{t m y}-R_{t m y} V_{t m x}}{R_{t m}^{2}} \\
R_{t m}=\sqrt{R_{t m x}{ }^{2}+R_{t m y}{ }^{2}} \\
V_{t m}=-\dot{R}=\frac{-\left(R_{t m x}{ }^{2}\right.}{\left.R^{m}+R_{t m y} V_{t m y}\right)} \\
R_{t m}
\end{array}\right\}
$$

\section{Gravity PN guidance law}

\section{A. Deducing process}

Based on the definition that orientation of overload of instruction of guidance law and orientation of line-of-sight are vertical, list TPN guidance law equation [3].

$N_{c}=V_{t m} \dot{q}$

Based on TPN guidance law, a gravity component is added, list the improved guidance law equation. $N_{c}=K V_{t m} \dot{q}+c g \cos \theta, \quad c>1$

Combine (2) and (4), list the gravity PN guidance law equation.

$N_{c}=K V_{t m} \dot{q}+c g \cos \theta=K \frac{-\left(R_{t m x} V_{t m y}+R_{t m y} V_{t m y}\right)\left(R_{t m x} V_{t m x}-R_{t m y} V_{t m y}\right)}{R_{t m}^{3}}+c g \cos \theta$

\section{B. Simulation}

Coordinate of guided-bomb is $(0,1000)$, coordinate of target is $(5000,0)$, initial velocity of guided-bomb is $v=0.6 M a$, parameter $c$ choose 1 and 2 and 3 , simulation results is below.

The results demonstrate that the landing error is acceptable and that the falling angle can't be controlled. Increasing compensate parameter $c$ will also increase falling angle and overload of instruction of guidance law. Excessive overload results in increased landing error and may lead to out of control of guided-bomb. So, gravity PN guidance law is suitable for situation rather in need of low landing error than in need of precise angle constraint.
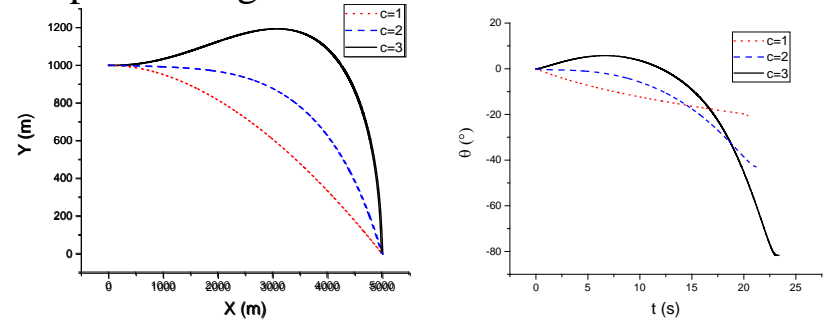

Fig. 2 Ballistic curve Fig. 3 Path angle of guided-bomb curve

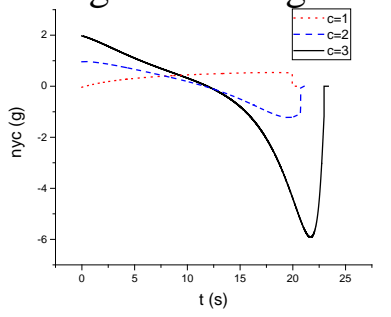

Fig. 4 Overload curve 
Table 1 Three Scheme comparing

\begin{tabular}{ccc}
\hline $\mathrm{U}$ & Landing error(m) & Falling angle $^{\circ}$ ) \\
\hline 1 & 0.55 & -20.2 \\
2 & 0.65 & -42.5 \\
3 & 0.78 & -81.4 \\
\hline
\end{tabular}

\section{Bias proportional navigation guidance law}

\section{A. Deducing process}

Based on the definition, list Bias proportional navigation guidance law equation [4].

$n_{y c}=K V_{m} \dot{q}+\varepsilon$

Here, $\varepsilon$ refers to bias component, based on the definition, list another form of (6).

$V_{m} \dot{\theta}_{m}=N \dot{q}+\varepsilon$

Based on the definition of differential coefficient, list another form of (7).

$V_{m} \frac{\theta_{m 1}-\theta_{m 0}}{\Delta t}=K \dot{q} \frac{q_{1}-q_{0}}{\Delta t}+\varepsilon$

Here, subscript 0 and 1 refers to initial time and terminal time of $\Delta t$. When 1 refers to the time target and guided-bomb meet, accordingly, $\Delta t$ refers to remnant flight time $t_{g o}$, list equation.

$V_{m} \frac{\theta_{m f}-\theta_{m 0}}{t_{g o}}=K \dot{q} \frac{q_{f}-q_{0}}{t_{g o}}+\varepsilon$

Here, $\theta_{m f}$ refers to path angle of guided-bomb, $q_{f}$ to line-of-sight angle.

At the terminal time $t_{f}, r\left(t_{f}\right)=0$,simply (9).

$V_{t} \sin q_{f}=V_{m} \sin \left(q_{f}-\theta_{m f}\right)$

)

Unfold (10), list equation of $q_{f}$.

$q_{f}=\arctan \frac{V_{m} \sin \theta_{m f}}{V_{m} \cos \theta_{m f}-V_{t}}$

)

Combine (11) and (9). List equation of $\varepsilon$.

$\varepsilon=\frac{V_{m}\left(\theta_{m f}-\theta_{m 0}\right)-K \dot{q}\left(\arctan \frac{V_{m} \sin \theta_{m f}}{V_{m} \cos \theta_{m f}-V_{t}}-q_{0}\right)}{t_{g o}}$

)

List equation of bias proportional guidance law.

$n_{y c}=K \dot{q}+\frac{V_{m}\left(\theta_{m f}-\theta_{m 0}\right)-N\left(\arctan \frac{V_{m} \sin \theta_{m f}}{V_{m} \cos \theta_{m f}-V_{t}}-q_{0}\right)}{\operatorname{tgo}}$

)

Transfer (13) into general form.

$n_{y c}=K \dot{q}+\frac{V_{m}\left(\theta_{m f}-\theta_{m}\right)-K \dot{q}\left(\arctan \frac{V_{m} \sin \theta_{m f}}{V_{m} \cos \theta_{m f}-V_{t}}-q\right)}{\operatorname{tgo}}$

)

If the target is standstill, simplify (14). 
$n_{y c}=K \dot{q}+\frac{V_{m}\left(\theta_{m f}-\theta_{m}\right)-K\left(\theta_{m f}-q\right)}{t_{g o}}$

)

Moreover, list equation of $t_{g o}$. $t_{g o}=r / V_{m}$

)

\section{B. Simulation}

Coordinate of guided-bomb is $(0,1000)$, coordinate of target is $(5000,0)$, velocity of guided-bomb is $v=0.6 \mathrm{Ma}$, parameter $\theta_{d}=-40^{\circ} 、 \theta_{d}=-50^{\circ} 、 \theta_{d}=-60^{\circ}$, simulation results is below.

The results demonstrate that the landing error is acceptable. Difference valve between expectation falling angle and true falling angle is $3^{\circ} \sim 4^{\circ}$. So, comparative to gravity PN guidance law, bias proportional guidance law's performance in angle constraint has been improved with space for further improvement.
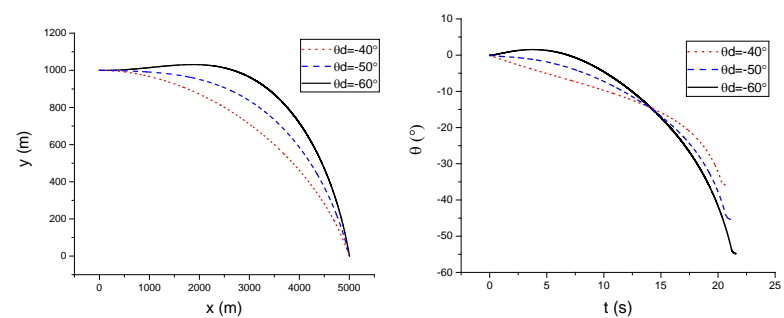

Fig. 5 Ballistic curve Fig. 6 Path angle of guided-bomb curve

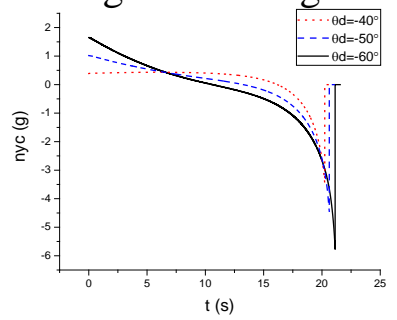

Fig. 7 Overload curve

Table 2 Three Scheme comparing

\begin{tabular}{ccc}
\hline$\theta_{d}\left({ }^{\circ}\right)$ & Landing error(m) & Falling angle $\left({ }^{\circ}\right)$ \\
\hline-40 & 0.51 & -36.1 \\
-50 & 0.60 & -46.2 \\
-60 & 0.72 & -56.8 \\
\hline
\end{tabular}

\section{Sliding mode variable structure guidance law}

\section{A. Deducing process}

Based on the fact that steering instructions of guidance loop are all normal direction overload, so, assume that magnitude of velocity of guided-bomb is standstill, only orientation changed. List movement equation set of projectile body particle [5].

$$
\left.\begin{array}{c}
\dot{x}=V_{m} \cos \theta \\
\dot{y}=V_{m} \sin \theta \\
\dot{\theta}=\left(a_{y c}-g \cos \theta\right) / V_{m}
\end{array}\right\}
$$

)

Based on the second form in (1), make a derivation, combine it with (17). List the equation below. 
) $\ddot{q}=\frac{1}{R_{t m}}\left(-2 \dot{R}_{t m} \dot{q}+N_{t} \cos \eta_{t}-N_{c} \cos \eta\right)=\frac{1}{R_{t m}}\left(-2 \dot{R}_{t m} \dot{q}+N_{t} \cos \eta_{t}-\left(a_{y c}-g \cos \theta\right) \cos \eta\right)=\frac{1}{R_{t m}}\left(-2 \dot{R}_{t m} \dot{q}+N_{t} \cos \eta_{t}-a_{y c} \cos \eta+g \cos \theta \cos \eta\right)$

)

List state equation.

$\left.\begin{array}{c}\dot{x}_{1}=x_{2} \\ \left.\dot{x}_{2}=\frac{-2 \dot{R}_{m}}{R_{t m}} x_{2}+\frac{-\cos \eta}{R_{t m}} u+\frac{1}{R_{t m}}\left(N_{t} \cos \eta_{t}+g \cos \theta \cos \eta\right)\right)\end{array}\right\}$

)

Here, $x_{1}=q, x_{2}=\dot{q}, u=a_{y c}$.

Assume that $\theta_{d}$ refers to expectation falling angle, $\theta_{t}$ to path of angle when target and guided-bomb meet, $\Delta$ to falling angle constraint. List the equation below.

$\theta_{d}=\theta_{t}+\Delta$

)

Here, $\Delta= \pm \frac{\pi}{2}$ means guided-bomb attack target vertically, $\Delta= \pm \pi$ means rear-end attack or bow-on attack.

When target and guided-bomb infinitely approached, line-of-sight angle is $q_{\text {end }}$, overload of target and guided-bomb can be neglected. List the simplified equation.

$\left.\begin{array}{c}V_{t} \sin \left(q_{\text {end }}-\theta_{t}\right)=V_{m} \sin \left(q_{\text {end }}-\theta_{d}\right) \\ |q-\theta|<\frac{\pi}{2}\end{array}\right\}$

)

Based on the fact that target is standstill, so, $V_{t}=0, \theta_{t}=0$, combine (21) and (22).

$q_{\text {end }}=\theta_{d}=\Delta$

)

Based on the fact that target is standstill, so, $\theta_{d}=p$. Through constraint line-of-sight angle, falling angle constraint can be achieved. Sliding mode can be designed.

$s=\dot{q}+\frac{\lambda V_{m}}{R_{t m}}\left[q-q_{\text {end }}\right]=x_{2}+\frac{\lambda V_{m}}{R_{t m}}\left[x_{1}-q_{\text {end }}\right]$

)

State approaching sliding mode choose widely used exponential reaching law.

$\dot{s}=-\frac{k\left|\dot{R}_{t m}\right|}{R_{t m}} s-\frac{\varepsilon}{R_{t m}} \operatorname{sgn}(s)$

)

Here, $\operatorname{sgn}(s)$ is saturation function.

$\begin{cases}\operatorname{sgn}(s)>0 & s>0 \\ \operatorname{sgn}(s)=0 & s=0 \\ \operatorname{sgn}(s)<0 & s<0\end{cases}$ 
Combine (24) and (25).

$s \dot{s}=-\frac{k\left|\dot{R}_{t m}\right|}{R_{t m}} s^{2}-\frac{\varepsilon}{R_{t m}} \operatorname{sgn}(s) \cdot s$

)

Easy to judge that $s \dot{s}<0$. As a result, we can conclude that exponential reaching law satisfies index of existence and reachability and stability of sliding mode.

Differential (24), combine it with (20).

$a_{y c}=\left[\left(\lambda V_{m}+k\left|\dot{R}_{t m}\right|-2 \dot{R}_{t m}\right) \dot{q}+\lambda V_{m}\left(k\left|\dot{R}_{t m}\right|-\dot{R}_{t m}\right)\left(q-q_{e n d}\right) / r+\varepsilon \operatorname{sgn}(s)\right] / \cos \eta+g \cos \theta$

)

\section{B. Simulation}

Coordinate of guided-bomb is $(0,1000)$, coordinate of target is $(5000,0)$, velocity of guided-bomb is $v=0.6 M a$, parameter $\theta_{d}=-40^{\circ} 、 \theta_{d}=-50^{\circ} 、 \theta_{d}=-60^{\circ}$, simulation results is below.

The results demonstrate that sliding mode variable structure guidance law meet both index of low landing error and precise falling angle constraint, overload is far less than performance applied by gravity PN guidance law and bias proportional navigation guidance law.
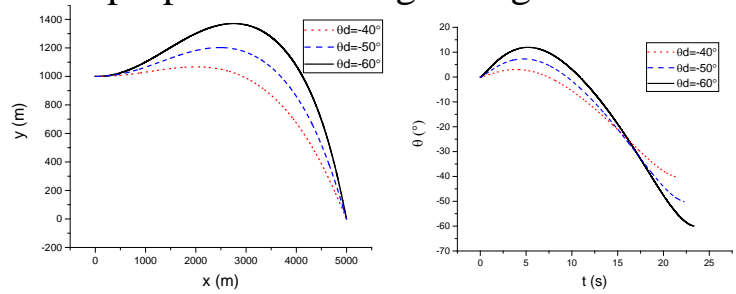

Fig. 8 Ballistic curve Fig. 9 Path angle of guided-bomb curve

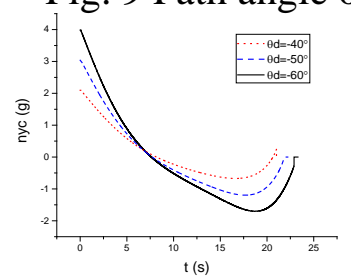

Fig. 10 Overload curve

Table 3 Three Scheme comparing

\begin{tabular}{ccc}
\hline$\theta_{d}\left({ }^{\circ}\right)$ & Landing error(m) & Falling angle $\left({ }^{\circ}\right)$ \\
\hline-40 & 0.48 & -40.04 \\
-50 & 0.51 & -50.02 \\
-60 & 0.68 & -60.03 \\
\hline
\end{tabular}

\section{Summary}

In this paper, the gravity PN guidance law, bias proportional navigation guidance law with terminal impact angle constraint and sliding mode variable structure guidance law with terminal impact angular constraint are all derived, simulated and analyzed in the terminal stage. The results demonstrate that gravity PN guidance law can simply guarantee index of landing error, bias navigation guidance law and sliding mode variable structure guidance law can guarantee both index of landing error and falling angle constraint. Moreover, performance of sliding mode variable guidance law is better than bias navigation guidance law.

\section{References}

[1] East, D.J. Some aspects of guidance loop design for SAM systems. Advanced Technology for SAM Systems Analysis Synthesis and Simulation (AGARD-LS-135), p 3/1-14, May 1984. 
[2] Murphy, C.H. Flight mechanics of an elastic symmetric missile. Journal of Guidance, Control, and Dynamics, v 24, n 6, p 1125-1132, November/December 2001.

[3] Chao-Yong Li, Wu-Xing Jing. Geometric approach to capture analysis of PN guidance law. Aerospace Science and Technology, v 12, n 2, p 177-83, March 2008

[4] Gao Feng, Tang Sheng-jing, Shi Jiao, Guo Jie. A bias proportional navigation guidance law based on terminal impact angle constraint. Transactions of Beijing Institute of Technology, v 34, n 3, p 277-82, March 2014.

[5] Wang, Hong-Qiang, Fang, Yang-Wang, Yin, Hong-Wu. Design of a sliding mode variable structure guidance law. Xi Tong Gong Cheng Yu Dian Zi Ji Shu/Systems Engineering and Electronics, v 31, n 10, p 2445-2449, October 2009. 